\title{
Administration of anaesthesia during drug-induced sleep endoscopy: implementation of a computer system to assist the decision on how to administer analgosedation
}

\author{
Nicoleta Dumitrescu, ${ }^{1,2}$, Codrut Sarafoleanu ${ }^{1,2}$, Bogdan Mihail Cobzeanu ${ }^{3}$ \\ ${ }^{1}$ ENT\&HNS Department, "Sfanta Maria” Hospital, Bucharest, Romania \\ 2"Carol Davila” University of Medicine and Pharmacy, Bucharest, Romania \\ ${ }^{3}$ Department of Otorhinolaryngology, "Grigore T. Popa" University of Medicine and Pharmacy, Iasi, Romania
}

\begin{abstract}
BACKGROUND. Starting from the European and American guidelines regarding sedation during gastrointestinal investigations and continuing with the American Society of Anesthesiologists (ASA) recommendations, we have developed a software that helps the ENT specialist to decide whether the patient can undergo a moderate sedation in the operating room, or in the outpatient office, during the drug-induced sleep endoscopy.

MATERIAL AND METHODS. The application is written in C\#, using the .NET Framework, and is available for the Windows operating system. The application has a very simple and user-friendly interface, and a bare-bones implementation, focusing strictly on the decision-making process.

RESULTS. The application involves the information obtained from patient history, clinical examination and polysomnography. After filling all the patient's data, it will show the result that can be one of the following: "treatment can be ambulatory", "treatment should be done in the hospital" or "treatment is not recommended".
\end{abstract}

CONCLUSION. The decision-making software application may substitute the evaluation of the anaesthesiologist during drug-induced sleep endoscopy in certain conditions.

KEYWORDS: drug-induced sleep endoscopy, analgosedation, software application.

\section{INTRODUCTION}

It is well known the great importance of sleep apnea in the occurrence of daytime sleepiness, mood changes and decrease in work capacity, as well as in the prevalence of cardiovascular disease and the increasing number of car accidents ${ }^{1,2}$. Therefore, the correct diagnosis has crucial importance and the medical investigation must illustrate a complete clinical image and also offer a complete evaluation of the anatomical regions of the superior respiratory system ${ }^{3}$.

Drug-induced sleep endoscopy is part of the exploring methods of sleep disorders. This involves inducing sleep using propofol administered progressively with a speed of $3 \mathrm{mg} / \mathrm{kg}$ bw/hour until a bispectral index of 65-75. Then, the flexible endoscope is introduced through the nasal fossa continuing through the pharynx and larynx. Therefore, it may be observed the anatomic aspect of the retropalatal region, retroglossal region and, also, the vibrating and obstructive regions. There are several studies that show links between sleep apnea severity and multilevel obstruction and authors who observed retropalatal obstruction more frequently than other obstruction regions $\mathrm{s}^{4,5}$.

During drug-induced sleep endoscopy certain side effects may occur, like prolonged apnea epi- 
Table 1. The sedation levels and their effect on vital parameters (according to the American Society for Gastrointestinal Endoscopy).

\begin{tabular}{ccccc}
\hline & Minimal sedation & Moderate sedation & Deep sedation & General anaesthesia \\
\hline Responsiveness & Normal response & Purposeful response & Purposeful response after repeated stimulation & Unarousable \\
\hline Airway & Unaffected & No intervention required & Intervention may be required & Intervention often required \\
\hline Spontaneous ventilation & Unaffected & Adequate & May be inadequate & Frequently inadequate \\
\hline Cardiovascular function & Unaffected & Maintained & Maintained & Impaired \\
\hline
\end{tabular}

sodes with critical oxygen desaturation, propofol allergy, bleeding, laryngospasm or aspiration syndrome, requiring immediate surgical intervention (haemostasis, tracheostomy) and/or endotracheal intubation. That is why it is particularly important to carefully select the patients who undergo this investigation.

The importance of this investigation is given by the possibility to observe, in real time, the collapsed anatomic areas, muscle hypotonia and further to evaluate and monitor certain types of treatment and to select more accurately the patients for a specific treatment ${ }^{6}$.

Regarding sedation, it represents the inhibition of the consciousness by administering proper sedative medication. According to the American Society of Anesthesiologists (ASA), there are four sedation levels according to the patient response to stimulation and the effect on the vital parameters ${ }^{7,8}$. While performing drug-induced sleep endoscopy, a moderate level of sedation is applied, which does not require the interven- tion of the anaesthesiologist on the respiratory tract because the spontaneous breathing and the cardiovascular function remain in proper parameters.

As far as the financial and time aspects go, the administration of sedation in the outpatient office, without anaesthetist, but in the presence of a qualified nurse, reflects in a significant reduction of costs ${ }^{9,10}$.

The objective of this study concerns the collaboration between the ENT specialist and the anaesthesiologist for administrating proper analgosedation during drug-induced sleep endoscopy (DISE). In this respect, we thought about a decision-making software application which may substitute the consultation of the anaesthesiologist during sleep endoscopy, in certain conditions.

\section{MATERIAL AND METHODS}

Starting from the European and American guidelines regarding sedation during gastrointesti-

Table 2. Clinical and anthropometric parameters which influence the sleep endoscopy recommendation.

\begin{tabular}{|c|c|c|c|}
\hline & Outpatient service & Hospital & Contraindication \\
\hline AHI (\#/h) & $<30$ & $30-70$ & $>70$ \\
\hline BMI (kg/m2) & $<30$ & $30-40$ & $>40$ \\
\hline Abdominal circumference $W(\mathrm{~cm})$ & $<80$ & $80-88$ & $\geq 88$ \\
\hline Abdominal circumference $M(\mathrm{~cm})$ & $<94$ & $94-102$ & $\geq 102$ \\
\hline Neck circumference W (cm) & $<33$ & $33-37$ & $>37$ \\
\hline Neck circumference $M(\mathrm{~cm})$ & $<36$ & $36-40$ & $>40$ \\
\hline Hypertension & Controlled & Not controlled & Not controlled \\
\hline Diabetes & Controlled & Not controlled & Not controlled \\
\hline Pulmonary pathology & Light & & \\
\hline Cardiac pathology & & $\begin{array}{l}\text { Not recent acute myocardial infarction, } \\
\text { stroke, transitory ischemic attack, } \\
\text { cardiac stent; light valvular disorder }\end{array}$ & $\begin{array}{l}\text { Recent acute myocardial infarction, } \\
\text { stroke, transitory ischemic attack, } \\
\text { cardiac stent; severe valvular disorder }\end{array}$ \\
\hline
\end{tabular}




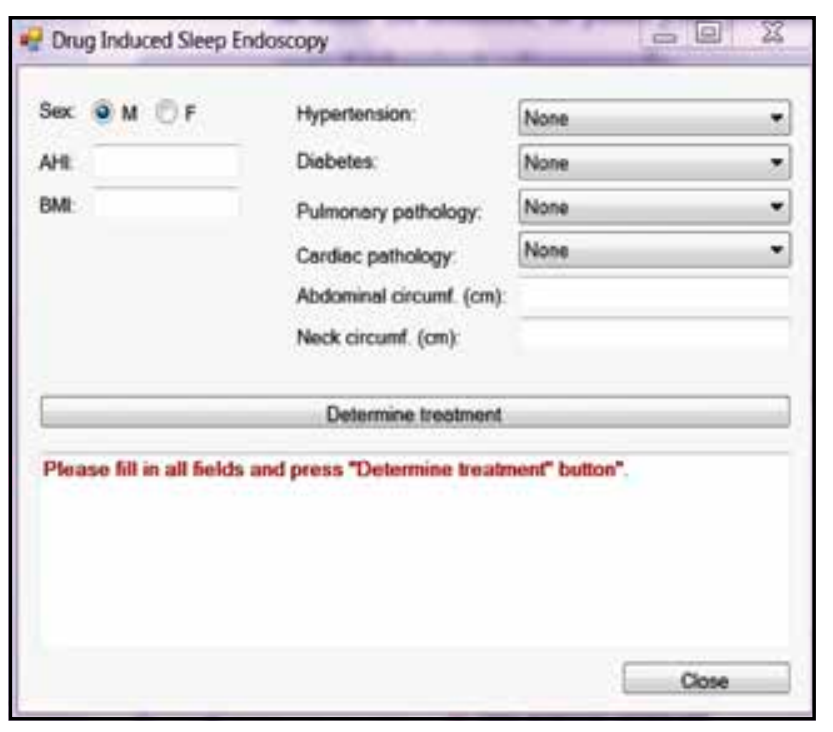

Figure 1. The application interface before the introduction of patient's data.

nal investigations (Table 1) and continuing with ASA recommendations, we have developed a software that helps the ENT specialist to decide whether the patient can undergo a moderate sedation in the operating room, or in the outpatient office. This decisional algorithm stood at the basis of creating a software to help the specialist when an anaesthesiologist cannot be consulted.

A small, simple computer application has been developed in order to exemplify the usage of the decision criteria described previously. The application is written in C\#, using the .NET Framework, and is available for the Windows operating system. In order to obtain a decision on how to proceed to treat the patient, the user is prompted to enter all the relevant details (namely the sex of the patient, apnea-hypopnea index (AHI), body mass index (BMI), abdominal and neck circumferences, presence of hypertension, diabetes, pulmonary or car-

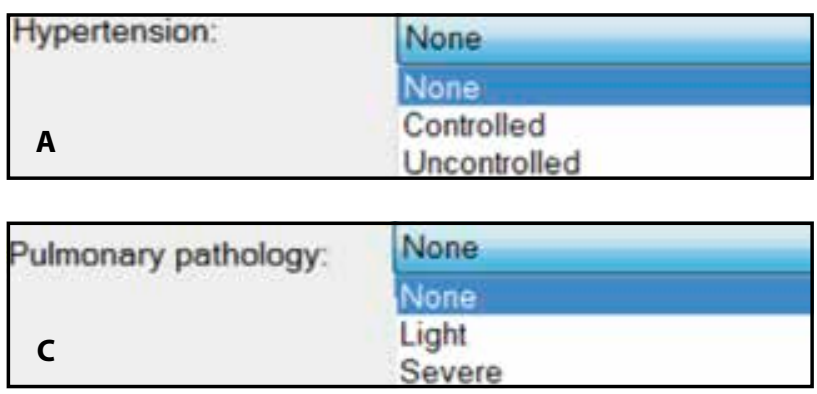

diac pathology), and then, using the decision chart previously described, the application determines how the treatment should proceed (Table 2).

The application has a very simple and userfriendly interface, and a bare-bones implementation, focusing strictly on the decision-making process. It is also portable, meaning it can be run on any Windows machine, whether from a local drive, or directly from a portable media (such as a USB stick), without requiring any installation process.

\section{RESULTS}

We have introduced the decision-making process of sedation in a simple computer application that can be used by the medical staff both in the hospital, as well as in the outpatient office. The application involves the information obtained from patient history, clinical exam and polysomnography.

In the left side of the window app, there are the blanks that must be filled in with the patient's sex, the apnea-hypopnea index value, and the body mass index value, as can be seen in Figure 1. In the right side of the window app, there are four blanks corresponding to the pathologies that interest the anaesthesiologists: blood pressure, diabetes, pulmonary and cardiological disorders (Figure 1). Therefore, the values of the glycaemia and the blood pressure must not be filled in the application, but one of the options "none", "controlled" or "not controlled" will be marked, as presented in Figure 2 a,b.

Regarding pulmonary pathology, it is important the grading of the disorder and not its type. Considering the patient's medical history, one of the following items can be selected: "none", "light" and "severe" (Figure 2c). With respect to the cardiological pathology, it is of interest the period that passed from the latest acute episode, regardless of the type of cardiovascular disorder and the
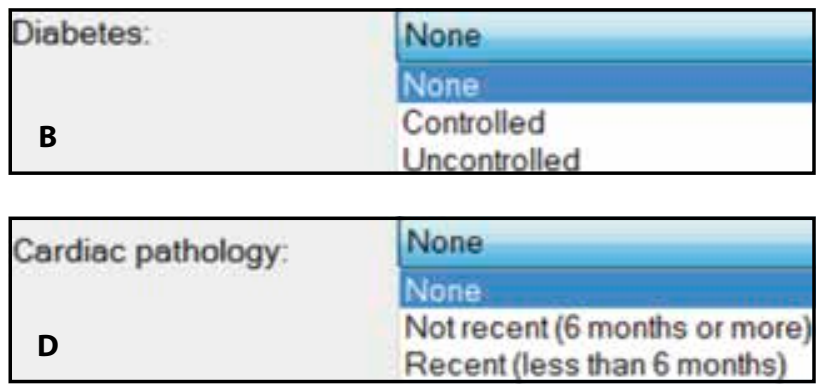

Figure 2. Exemplifying the blanks corresponding to the pathologies concerning the anaesthesiologists for the patient examination: (a) hypertension - absent or present controlled / uncontrolled; (b) diabetes, regardless its type - absent or present controlled / uncontrolled; (c) pulmonary pathology none, light or severe; (d) cardiac pathology - none, not recent (more than 6 months from the latest episode) or recent (less than 6 months from the latest episode). 


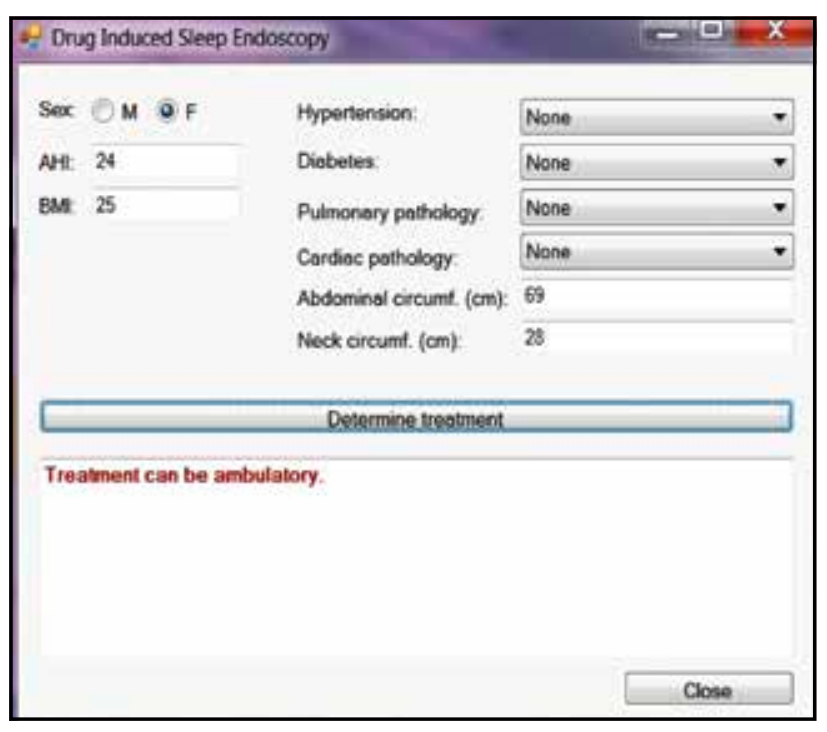

Figure 3. A case simulation for which DISE can be performed in the outpatient office by specially trained medical staff.

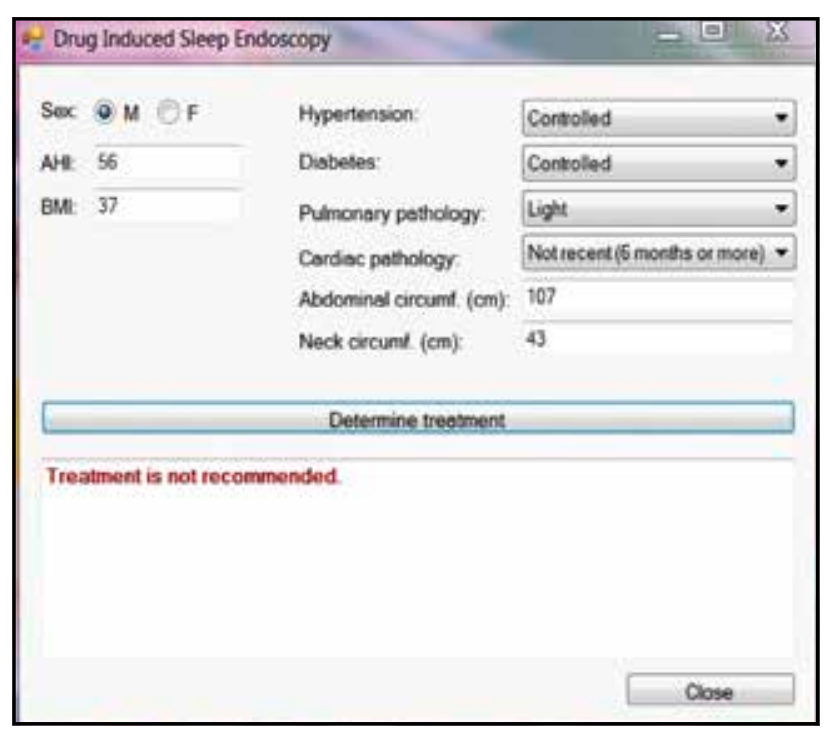

Figure 5. A case simulation for which DISE is not recommended for the high risk of sedation.

risk factors implied. Therefore, one of the following items can be selected: "none", "not recent more than 6 months from the latest acute episode", "recent - less than 6 months from the latest acute episode" (Figure 2d).

At the bottom of the app, there are two more blanks in which the values in centimetres of the abdominal and neck circumferences can be introduced.

After filling all the patient's data, the button that will start the computing engine and will show the result in the white screen from the bottom of the window is pressed. The result is written in red colour and can be one of the following: "treatment

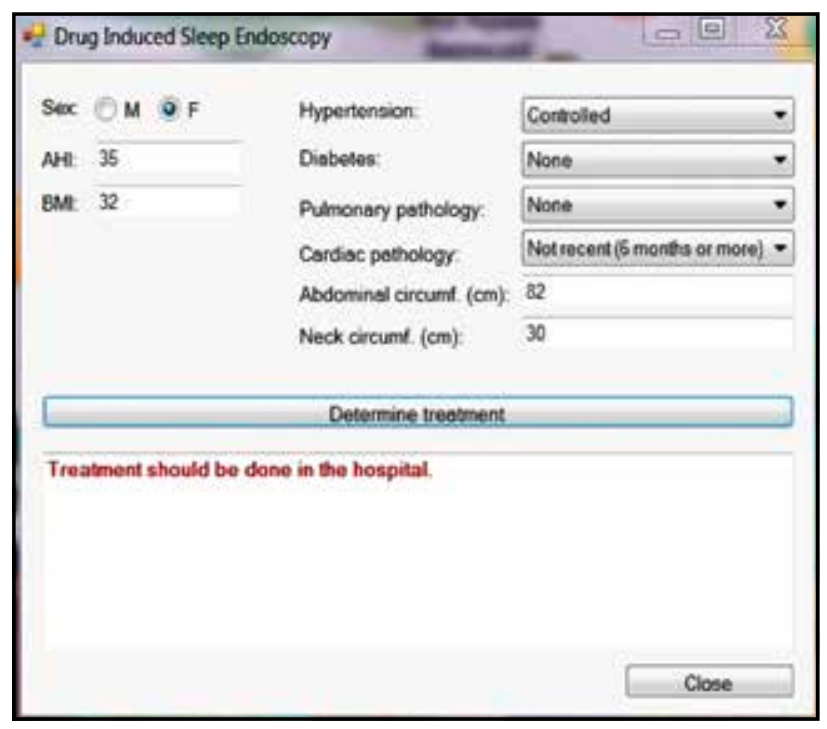

Figure 4. A case simulation for which DISE can be performed in the operating room in the presence of an anaesthesiologist.

can be ambulatory" (Figure 3), "treatment should be done in the hospital" (Figure 4) or "treatment is not recommended" (Figure 5).

\section{DISCUSSIONS}

We have brought into light the possibility of performing drug-induced sleep endoscopy in the outpatient office for those patients without anaesthesiologic risk according to the ASA criteria. Even though there is no consensus regarding analgosedation management for this clinical investigation, the American and European Societies of Gastroenterology released guidelines for sedation performed by trained nurses to act in critical situations ${ }^{8,10}$.

In order to perform drug-induced sleep endoscopy in the outpatient office, one must make sure that the facility and the medical team are prepared to undergo this procedure. The endoscopy room must have the monitoring equipment that comprises the assessment of heart rate, blood pressure, pulse-oximetry, electrocardiogram and ventilatory activity. The outpatient office must provide a recovery room for the patient ${ }^{11}$. During DISE and recovery time, there must be at least one member of the medical team trained to administer sedatives, monitor the patient, and manage airway or cardiovascular emergencies and, also, there must be equipment and medication at hand for resuscitation. The physician who performed the procedure must be present until the patient's discharge.

For lowering the risk of adverse pulmonary and cardiovascular events, the risk of aspiration must be assessed. To do so, the patient must consent for 
sedation and performing DISE and not to drink for 2 hours, respectively not to eat for 6 hours before the procedure ${ }^{12}$. The anamnesis shall provide information regarding allergies, previous sedation or anaesthesia, consumption of alcohol, drugs, medication or tobacco. A very important aspect regards the patient's history about pulmonary disease, cardiovascular or cerebral pathology and diabetes.

The physical exam aims at the features that may affect the sedation process and those that increase the risk for adverse events, respectively the Mallampati score, mouth opening, nasal cavity and anatomic aspect of the neck, oral cavity and mandible. Furthermore, the body mass index, the neck and abdominal circumferences and the polysomnographic results (apnea-hypopnea index) are very important decision factors in the sedation process. Moreover, the respiratory and cardiac assessment must be initially done with respect to heart rate, blood pressure and respiratory rate ${ }^{13}$.

The monitoring of the patient during drug-induced sleep endoscopy using propofol sedation regards heart rate, blood pressure, oxygen saturation, level of consciousness and respiratory rate. This assessment should be done before the procedure, immediately after the propofol administration, every 5 minutes during the procedure, after initial recovery and before discharge. It is recommended, for legal reasons, to record and archive all the patient's data.

The decision to discharge the patient should be accompanied by the interdiction, for at least 6 hours after procedure, to drive, drink alcohol, operate heavy machinery or involve in legal decisions ${ }^{14,15}$.

The concern of performing certain investigations and procedures in the outpatient office is a matter of research for many specialists due to patient compliance, cost reduction and time saving. Kiaer et al., in a 200-patient study, successfully performed sleep endoscopy using Propofol sedation without the presence of an anaesthesiologist, in normal conditions and with no side effects ${ }^{16}$.

The meta-analyses of the trials that compared propofol sedation administered by an anaesthesiologist with that administered by specialized nurses reported similar safety and similar cardiopulmonary events and, in addition, propofol was associated with better sedation and shorter recovery time ${ }^{17,18}$. There are also studies involving a large number of patients that found low incidence of propofol-related complications, a rigorous patient selection lowering the risk of adverse events during drug-induced sleep endoscopy ${ }^{19,20}$.

In this field of interest, the perspective may be targeted through organizing training classes for the nurses, which may further allow to safely perform drug-induced sleep endoscopy without the presence of an anaesthesiologist.

\section{CONCLUSIONS}

Artificial intelligence, software applications and robotic engineering are becoming more and more useful for both doctor and patient, they save not only lives, but also time and money.

The software application we have developed helps the ENT physician to decide safely and fast about the conditions in which the sleep endoscopy may take place in order to obtain good results without side effects.

Acknowledgements: I would like to thank Eng. Catalina Ioan for her support in developing the software application.

Conflict of interest: The authors declare there is no conflict of interest.

Contribution of authors: All the authors have equally contributed to this work.

\section{REFERENCES}

1. Shamsuzzaman ASM, Gersh BJ, Somers VK. Obstructive sleep apnea, implications for cardiac and vascular disease. JAMA. 2003;290(14):1906-14. DOI: 10.1001/jama.290.14.1906.

2. George CF, Smiley A. Sleep apnea \& automobile crashes. Sleep.1999;22(6):790-5.

3. Passali FM, Marzetti A, Bellussi L, Passali D. The diagnosis of obstructive sleep apnea syndrome. Romanian Journal of Rhinology. 2013;3(12):195-202.

4. Lo YL, Ni YL, Wang TY, Lin TY, Li HY, White DP, et al. Bispectral index in evaluating effects of sedation depth on drug-induced sleep endoscopy. Clin Sleep Med. 2015;11(9):1011-20.

5. De Corso E, Fiorita A, Rizzotto G, Mennuni GF, Meucci D, Giuliani $\mathrm{M}$, et al. The role of drug-induced sleep endoscopy in the diagnosis and management of obstructive sleep apnoea syndrome: our personal experience. Acta Otorhinolaryngol Ital. 2013;33(6):405-13.

6. Civelek S, Emre IE, Dizdar D, Cuhadaroglu C, Eksioglu BK, Eraslan AK, et al. Comparison of conventional continuous positive airway pressure to continuous positive airway pressure titration performed with sleep endoscopy. Laryngoscope. 2012;122(3):691-5. DOI 10.1002/lary.22494

7. An Updated Report by the American Society of Anesthesiologists Task Force on Sedation and Analgesia by Non-Anesthesiologists; Practice Guidelines for Sedation and Analgesia by Non-Anesthesiologists. Anesthesiology. 2002;96:1004-17. DOI: 10.1097/00000542 200204000-00031.

8. Early DS, Lightdale JR, Vargo JJ, Acosta RD, Chandrasekhara V, Chathadi KV, et al. Guidelines for sedation and anesthesia in GI endoscopy. Giejournal. 2018;87(2):327-37.

9. Hassan C, Rex DK, Cooper GS, Benamouzig R. Endoscopist-directed propofol administration versus anesthesiologist assistance for colorectal cancer screening: a cost-effectiveness analysis. Endoscopy. 2012;44(5):456-64. DOI: 10.1055/s-0032-1308936.

10. Dumonceau JM, Riphaus A, Schreiber F, Vilmann P, Beilenhoff U, Aparicio JR, et al. Non-anesthesiologist administration of propofol for gastrointestinal endoscopy: European Society of Gastrointestinal Endoscopy, European Society of Gastroenterology and Endoscopy 
Nurses and Associates Guideline-Update June 2015. Endoscopy. 2015;47(12):1175-89. DOI: 10.1055/s-0034-1393414.

11. Calderwood AH, Chapman FJ, Cohen J, Cohen LB, Collins J, Day LW, et al. Guidelines for safety in the gastrointestinal endoscopy unit. Gastrointest Endosc. 2014;79(3):363-72. DOI: 10.1016/j,gie.2013.12.015.

12. American Society of Anesthesiologists Committee. Practice guidelines for preoperative fasting and the use of pharmacologic agents to reduce the risk of pulmonary aspiration: application to healthy patients undergoing elective procedures: an updated report by the American Society of Anesthesiologists Committee on Standards and Practice Parameters. Anesthesiology. 2011;114(3):495-511. DOI: 10.1097/ALN.0b013e3181fcbfd9.

13. Vargo JJ, DeLegge MH, Feld AD, Gerstenberger PD, Kwo PY, Lightdale JR, et al. Multisociety sedation curriculum for gastrointestinal endoscopy. Gastrointest Endosc. 2012;76(1):e1-25.

14. Willey J, Vargo JJ, Connor JT, Dumot JA, Conwell DL, Zuccaro G. Quantitative assessment of psychomotor recovery after sedation and analgesia for outpatient EGD. Gastrointest Endosc. 2002;56(6):8106. DOI: $10.1067 / \mathrm{mge} .2002 .129609$.

15. Horiuchi A, Nakayama Y, Hidaka N, Ichise Y, Kajiyama M, Tanaka N. Low-dose propofol sedation for diagnostic esophagogastroduodenoscopy: results in 10,662 adults. Am J Gastroenterol. 2009;104(7):1650-5. DOI: 10.1038/ajg.2009.250.
16. Kiaer EK, Tønnesen P, Sørensen HB, Rubek N, Hammering A, Møller C, Hildebrandt AM, et al. Propofol sedation in Drug Induced Sedation Endoscopy without an anaesthesiologist - a study of safety and feasibility. Rhinology. 2019;57(2):125-131. DOI: 10.4193/ Rhin 18.066.

17. Goudra BG, Singh PM, Gouda G, Borle A, Gouda D, Dravida A, et al. Safety of non-anesthesia provider administered propofol (NAAP) sedation in advanced gastrointestinal endoscopic procedures: comparative meta-analysis of pooled results. Dig Dis Sci. 2015;60 (9):261227. DOI: $10.1007 / \mathrm{s} 10620-015-3608-x$.

18. Sethi S, Wadhwa V, Thaker A, Chuttani R, Pleskow DK, Barnett SR, et al. Propofol versus traditional sedative agents for advanced endoscopic procedures: a meta-analysis. Dig Endosc. 2014;26(4):515-24. DOI: $10.1111 /$ den.12219.

19. Sieg A, bng-Study-Group; Beck S, Scholl SG, Heil FZ, Gotthardt DN, et al. Safety analysis of endoscopist-directed propofol sedation: a prospective, national multicenter study of 24441 patients in German outpatient practices. J Gastroenterol Hepatol. 2014;29(3):517-23. DOI: $10.1111 /$ jgh.12458.

20. Gupta A. Preoperative screening and risk assessment in the ambulatory surgery patient. Curr Opin Anaesthesiol. 2009;22(6):705-11. DOI: 10.1097/ACO.0b013e3283301fb3. 